\title{
A Novel Registration Method for SAR and SPOT Images
}

\author{
Lixia Shu, Tieniu Tan, Ming Tang, Chunhong Pan \\ National Laboratory of Pattern Recognition, Institute of Automation, \\ Chinese Academy of Science, P.O. Box 2728, 100080 Beijing, P. R. China \\ e-mail: \{lxshu, tnt, tangm, chpan\}@nlpr.ia.ac.cn
}

\begin{abstract}
In this paper, we propose a novel mutual information (MI) based method to register SAR and SPOT images. The traditional MI can register SAR and SPOT images well. However, its robustness is weakened by the absence of orientation information. In our approach, we first extract orientation information at four directions by Gabor fliters, then MI of each corresponding image pair is calculated and the average value of $\mathrm{MI}$ is used as an improved measure for MI. Experiments show that our method is more robust than the traditional MI method. Meanwhile our method maintains comparable accuracy to the traditional MI, which is much better than coarse manual registration.
\end{abstract} filter

Keywords-image registation; mutual information; Gabor

\section{INTRODUCTION}

Image registration is the process of establishing pointby-point correspondence between two images obtained from a same scene. This process is very useful in remote sensing, medicine, cartography, computer vision, etc.

Many image registration methods have been proposed over the years [1]. In a whole, these methods can be classified into two categories: feature based methods and intensity based methods. Due to the difference of imaging condition between SAR and SPOT images, it is very difficult to extract corresponding features from them. Moreover, abundant speckle noise existing in SAR image aggravates this problem. Mutual information (MI), which belongs to intensity based methods, has been proved to be well suitable for multimodal medical image registration. Although MI is intensively researched in medical imaging, it is just recent that it comes to be used in remote sensing image registration [2-4]. Up to now, few papers use MI to register SAR and SPOT images. In this paper, we find that MI can also register SAR and SPOT image well, but the absence of orientation information reduces the robustness of the measure. To overcome this drawback, we adjust the MI measure by integrating orientation information of two images. First, Gabor filters are applied to filter SAR and SPOT images at four directions, then MI between the filtered images at corresponding direction is measured respectively, and the average value of $\mathrm{MI}$ is used as the new MI measure. Compared to the traditional MI based method, this new measure can greatly reduce MI artifacts, so it is less sensitive to initial point and can obtain larger attraction range. These properties make the registration function more robust. Meanwhile, our method maintains comparable accuracy to the traditional MI, which is much better than coarse manual registration.

\section{SAR AND SPOT IMAGE REGISTRATION BASED ON MI WITH ORIENTATION INFORMATION}

\section{A. Mutual Information}

Assume $A$ and $B$ denote two images, their MI is defined as

$$
I(A, B)=H(A)+H(B)-H(A, B)
$$

The items in the right hand of Eq.(1) are calculated respectively by [4]

$$
\begin{gathered}
H(A)=\sum_{a}-P_{A}(a) \log P_{A}(a), \\
H(B)=\sum_{a}-P_{B}(b) \log P_{B}(b), \\
H(A, B)=\sum_{a}-P_{A, B}(a, b) \log P_{A, B}(a, b) .
\end{gathered}
$$

where $H(A)$ and $H(B)$ are the entropies of $A$ and $B, H(A, B)$ is their joint entropy, $P_{A}(a)$ and $P_{B}(b)$ are the marginal probability mass functions, and $P_{A, B}(a, b)$ is the joint probability mass function, $a$ and $b$ denote two pixel values in A and B respectively. Suppose $h$ is the joint histogram of this image pair, these probability mass functions can be computed by [4]

$$
\begin{gathered}
P_{A, B}(a, b)=\frac{h(a, b)}{\sum_{a, b} h(a, b)}, \\
P_{A}(a)=\sum_{b} P_{A, B}(a, b), \\
P_{B}(a)=\sum_{a} P_{A, B}(a, b) .
\end{gathered}
$$

From the above formulas, we can see that the joint histogram $h$ determines the MI between two images. Given the intensity information in $A$, essentially, $H(A, B)$ estimates the uncertainty of the intensity information and $I(A, B)$ estimates the decrease of uncertainty at the corresponding location in $B$. That is to say, MI can be used to measure the statistical dependency between two images or to predict information of $\mathrm{B}$ from $\mathrm{A}$. The traditional MI based registration criterion shows that the two images should be registered when $\mathrm{I}(\mathrm{A}, \mathrm{B})$ is maximal.

This work is partly supported by the National 973 Program under Grant 2004 CB 318100 


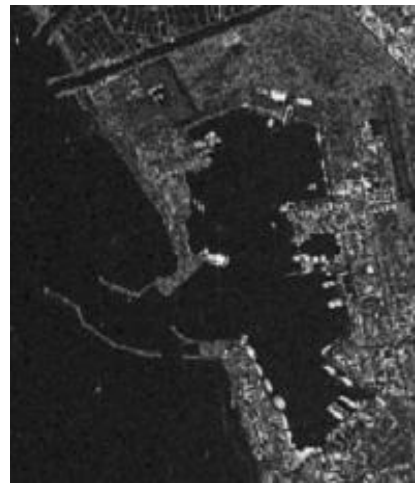

(a)

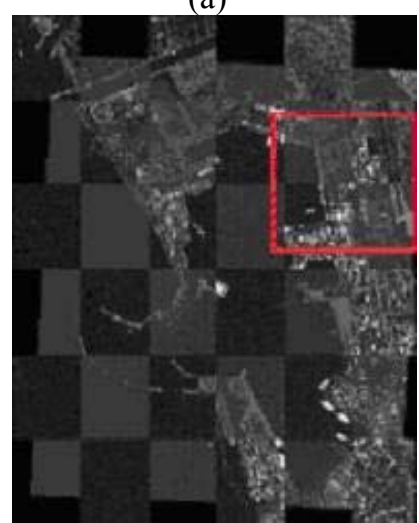

(e)

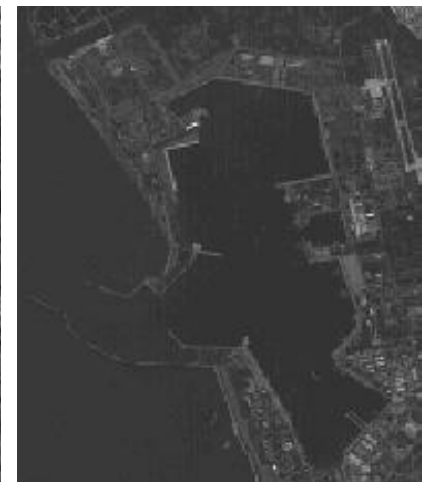

(b)

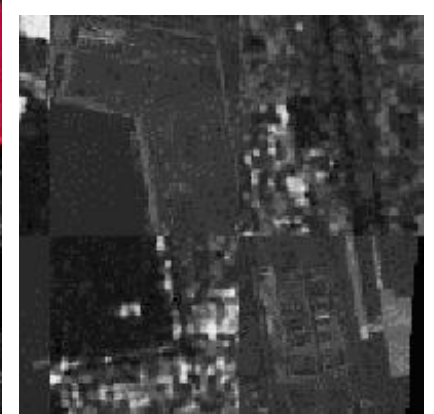

(f)

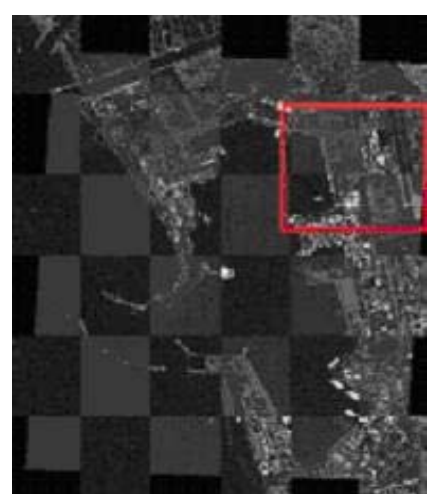

(c)

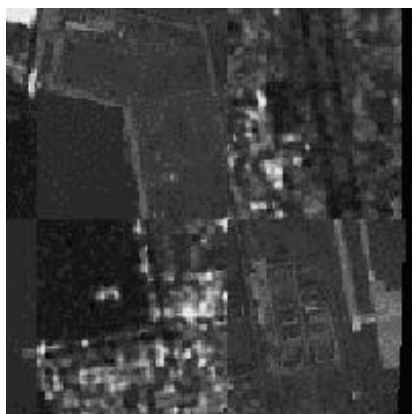

(g)

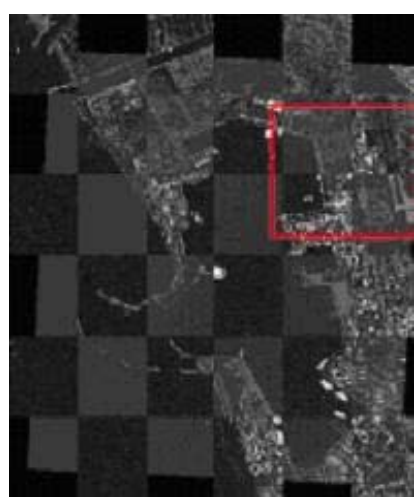

(d)

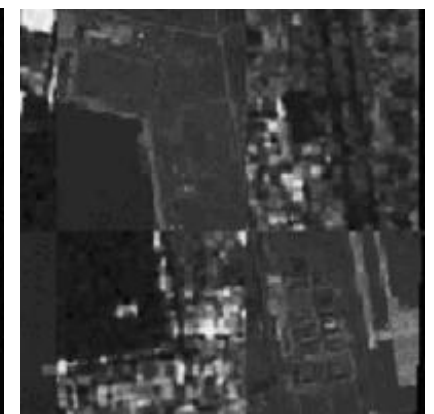

(h)

Fig.1. Explanation of registration accuracy; (a) SAR image A; (b) SPOT image B; (c) chessboard overlay of A and B after coarse manual registration; (d) chessboard overlay of A and B after the traditional MI based fine registration; (e) chessboard overlay of A and B after MIOI based fine registration; (f) close-up of the boxed region of (c), (g) close-up of the boxed region of (d), (h) close-up of the boxed region of (e).

Considering the sensitivity of MI measure to the amount of overlap between the images, normalized MI measure $\mathrm{NI}(A, B)=(\mathrm{H}(A)+\mathrm{H}(B)) / \mathrm{H}(A, B)[5]$ is chosen in our approach.

\section{B. MI with Orientation Information (MIOI)}

It is well known that 2D Gabor function is a Gaussianshaped function (known as envelop) modulated by a complex sinusoid (known as carrier) [6]

$$
\mathrm{h}(\mathrm{x}, \mathrm{y})=\mathrm{g}\left(\mathrm{x}^{\prime}, \mathrm{y}^{\prime}\right) \exp \left(2 \pi j F x^{\prime}\right)
$$

where

$$
\begin{gathered}
x^{\prime}=x \cos \theta+y \sin \theta, \\
y^{\prime}=-x \sin \theta+y \cos \theta, \\
\mathrm{g}\left(\mathrm{x}^{\prime}, \mathrm{y}^{\prime}\right)=\frac{1}{2 \pi \sigma_{x} \sigma_{y}} \exp \left[-\frac{1}{2}\left(\left(\frac{\mathrm{x}^{\prime}}{\sigma_{x}}\right)^{2}+\left(\frac{\mathrm{y}^{\prime}}{\sigma_{y}}\right)^{2}\right)\right] .
\end{gathered}
$$

Parameters $\theta$ and $\left(\sigma_{x}, \sigma_{y}\right)$ respectively define the rotation angel and the two axis scales of the Gaussian envelop. $F$ defines the spatial frequency of the sinusoid. Research shows that Gabor functions fit very well the receptive field weight functions in cortex cells [7]. The parameters of Gabor function directly capture the chief neurophysiological properties of spatial dimensions (determined by $\left.\left(\sigma_{x}, \sigma_{y}\right)\right)$, preferred orientation (determined by $\theta$ ), preferred spatial frequency (determined by $F$ ), and the tuning bandwidths for orientation and spatial frequency (determined jointly by $\theta$, $\left(\sigma_{x}, \sigma_{y}\right), F$ ) (extracted from [7]). By selecting proper parameters, a band filter with arbitrary orientation selectivity and arbitrary bandwidth can be produced. Image information at directed orientation can be extracted using this filter.

According to the definition of MI, we can see that it is completely determined by joint intensity histogram of two images, and no image orientation information is included in it. To solve this problem, by choosing proper parameters, we first produce a family of Gabor filters with different orientation selectivity, and use them to filter the two registered images at every direction. MI of each filtered image pair is measured. Apparently, it contains the corresponding direction information. The average value of MI at all directions is then used as an improved MI measure for registration. Orientation information at these chosen directions is thus included in the new MI measure.

Considering the computational cost, in this paper, only four directions are selected, which are $\theta=0, \theta=45^{\circ}, \theta=90^{\circ}$, $\theta=135^{\circ}$. Values of other parameters are $\sigma_{x}=3, \sigma_{y}=3.34$, $F=64$. 


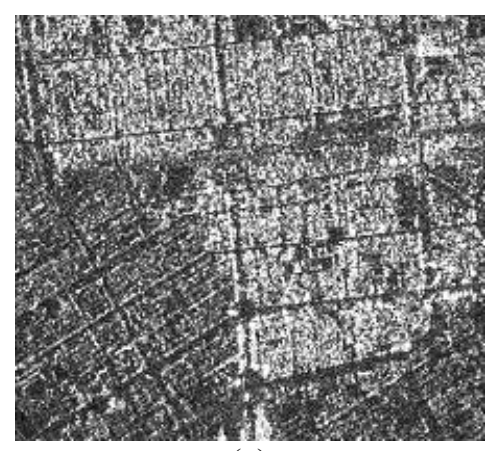

(a)

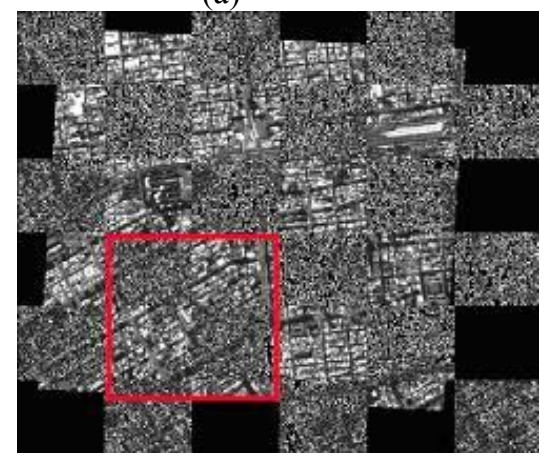

(e)

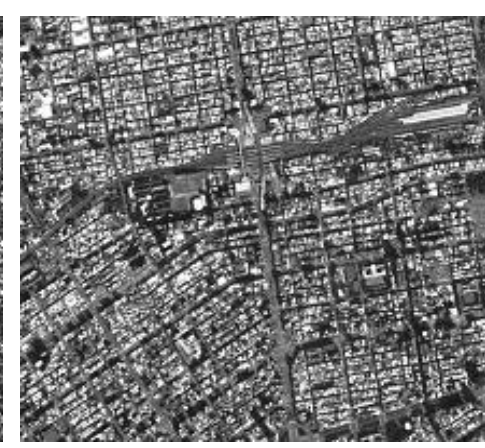

(b)

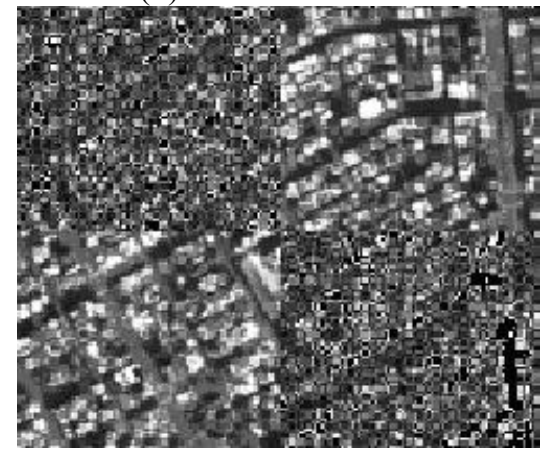

(f)

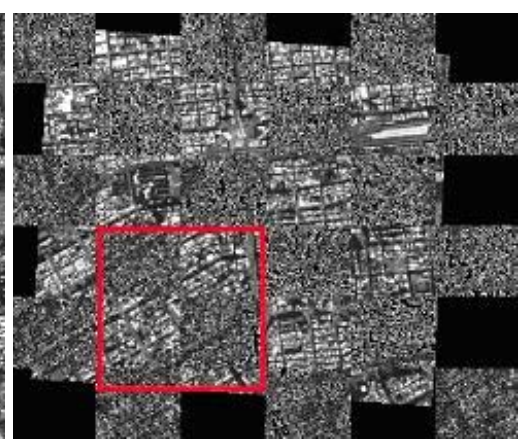

(c)

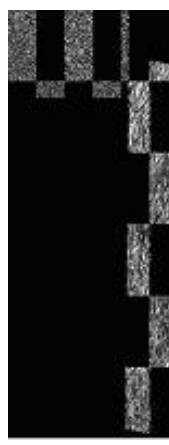

(d)

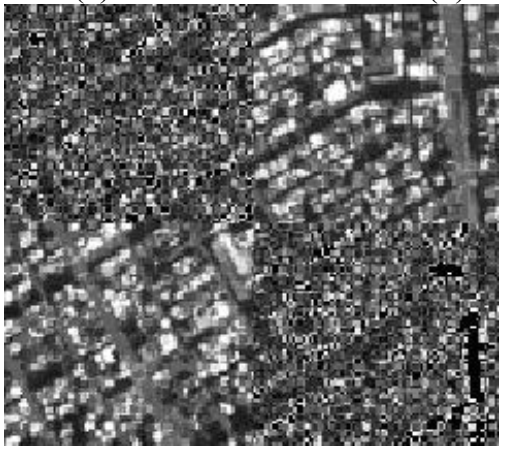

(g)

Fig. 2. Explanation of registration robustness ;(a) SAR image A; (b) SPOT image B; (c) chessboard overlay of A and B after coarse manual registration; (d) chessboard overlay of A and B after the traditional MI based fine registration; (e) chessboard overlay of A and B after MIOI based fine registration; (f) close-up of the boxed region of (c) ; (g) close-up of the boxed region of $(\mathrm{e})$
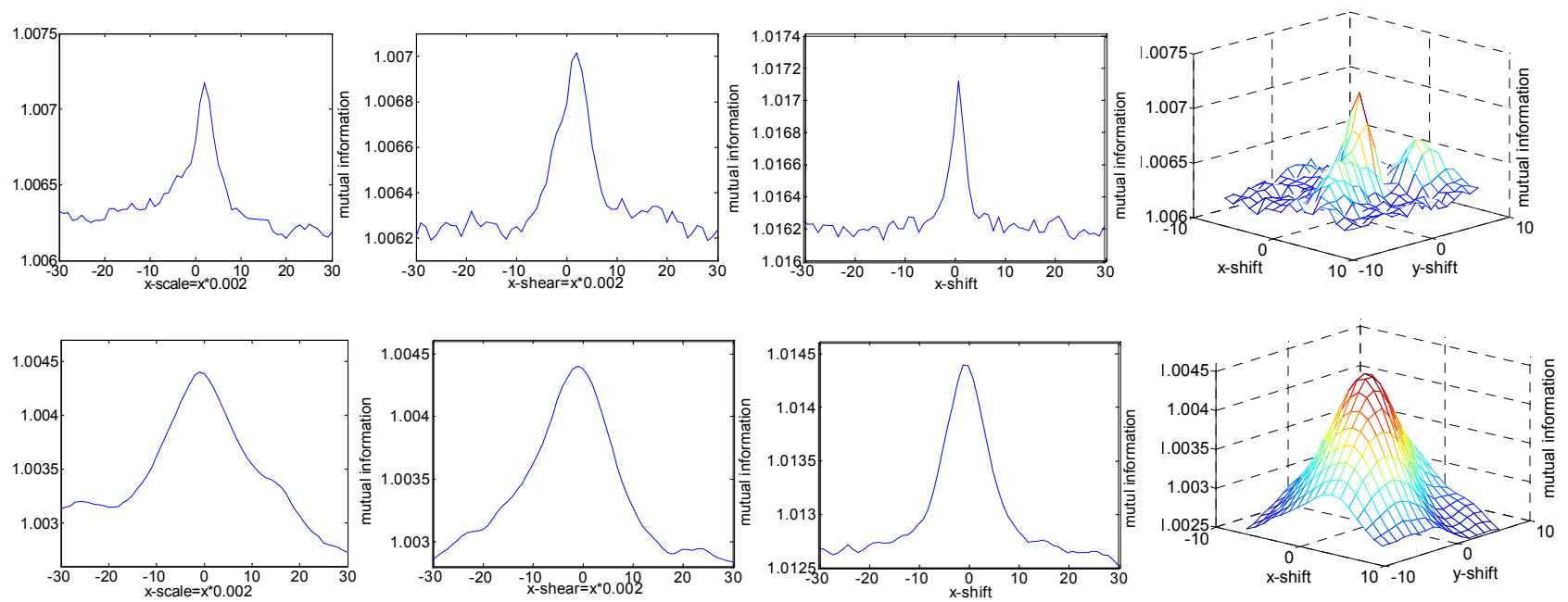

Fig.3. Registration function in Fig 2; the $1^{\text {st }}$ row: the traditional MI; the $2^{\text {nd }}$ row: MIOI; the $1^{\text {st }}$ to the $3^{\text {rd }}$ column: MI curves while parameters of $x$-scale, $x$-shear, $x$-shift changing; the $4^{\text {th }}$ column: MI surface while $x$-shift and $y$-shift changing

\section{EXPERIMENTS}

As indicated in [8], maximizing MI may not necessarily produce an optimal solution when the deformable transform is too flexible. So in our application, we apply coarse-to-fine method. Based on the manually chosen tie-points, the transform parameters between the two images are first estimated using the linear least-square technique. Then by using this coarse registration result as the initial point for optimization and MIOI as the fine registration function, the SAR and SPOT images are 
finely registered. Partial volume (PV) [9] and Powell method [10] are used as the interpolation and optimization method respectively. Affine transform is considered and six parameters, namely scale, shear, shift along $\mathrm{x}$ and $\mathrm{y}$ axes, are used.

Fig. 1 shows the registration accuracy of our method. (a) is a $526 \times 711$ Radarsat SAR sub-image acquired on Nov.2, 2000, and (b) is a $1180 \times 1530$ SPOT-5 sub-image acquired on Dec.31, 2002. By visual comparison, we can note that the registration of the MIOI based method is much better than the coarse manually method. To do a numerical comparison, we manually locate 16 pairs of check points with a good distribution and evaluate the registration accuracies (RMSE). The RMSE values are 5.3946 pixels for coarse registration and 3.1325 pixels for MIOI based fine registration. This indicates that the registration accuracy is greatly improved by our method. As to the traditional MI method, its RMSE is 3.2742 pixels, which is similar to that of our method.

Fig. 2 demonstrates the robustness of our method. Different from the experiment before-mentioned, the SAR image is not filtered. (a) and (b) are a pair of Radarsat SAR and SPOT-5 sub-images with size $468 \times 397$ and $882 \times 800$ that are cropped from the same source data as Fig 1 respectively. By visual comparison, we can note that traditional MI based method results in misregistration while MIOI based method yields satisfying result. The cause is clearly visible in Fig. 3. The figure shows that many artifacts exit in the traditional MI registration functions. Due to the artifacts, it tends to be trapped in local maximum if the initial point is not near the registration result. However, the inclusion of orientation information remarkably reduces the artifacts. MIOI registration function is smooth, which makes optimization easier to find out correct result even if the initial point is a little far away. That is the reason why the traditional MI based method fails while MIOI based method succeeds in this experiment. So compared to the traditional MI based method, our method is less sensitive to initial point for optimization, and can obtain larger attraction range. Thus, the proposed method is more robust. Meanwhile, in this experiment, one can also note that our method can work very well even if the SAR image is not filtered. This indicates that our method is robust to speckle noise, which outperforms many other registration methods.

\section{CONCLUSION}

In this paper, we have presented a novel MI based method to register SAR and SPOT images. The traditional MI is illdefined for orientation information is absent. In order to overcome this drawback, similarity between the filtered image pair which contains direction information extracted by Gabor function is evaluated by MI. The average value of MI is used as a new MI measure for registration. Experiments demonstrate that our method yields a better registration function than the traditional MI based method.

Compared to feature based methods, our method does not require that the registered images have specific features. Moreover, the SAR and SPOT images need not to be preprocessed. It is suitable for such images with following properties: 1) The structures in both images are similar. 2) The co-occurrence of the most probable values in the two images is maximized at registration.3) The information contained in each image is abundant enough.

However, because the size of remote sensing image is always very large, MI based method is time consuming. This problem is especially serious for the MIOI based method. Our future work will focus on how to decrease the computation and speed up the method. Moreover, it is also desirable to consider how to choose better orientations instead of the simple four directions used in this paper.

\section{REFERENCES}

[1] B. Zitova, and J. Flusser, " Image registration methods: a survey", Image and Vision Computing, vol. 21, no. 11, pp. 977-1000, 2003.

[2] A. A. Cole-Rhodes, K.L. Johnson, J. LeMoigne, and I. Zavorin, "Multiresolution registration of remote sensing imagery by optimization of mutual information using a stochastic gradient", IEEE Trans. Image Processing, Vol. 12, No. 12, pp. 1495-1511,2003.

[3] R. Fransens, C. Strecha, and L.V. Gool,“ Multimodal and multiband image registration using mutual information", http://earth.esa.int/rtd/Events/ESA-EUSC 2004/Ar24 Strecha.pdf

[4] H.M. Chen, P.K.Varshney, and M.K. Arora, “ Performance of mutual information similarity measure for registration of multitemporal remote sensing images", IEEE Tran. Geoscience and Remote Sensing, vol. 41, no. 11 , pp. 2445-2454, 2003.

[5] C. Studholme, D.L.G. Hill, and D.J. Hawkes, “ An overlap invariant entropy measure of 3D medical image alignment", Pattern Recognition, vol. 32, no. 1, pp.71-86, 1999

[6] Javier R. Movellan. “ Tutorial on gabor filters", http://mplab.ucsd.edu/ tutorials/pdfs/Gabor.pdf

[7] J.Daugman. " Complete discrete 2-D Gabor transform by neural networks for image analysis and compression", IEEE Trans .ASSP,vol.36, no. 7, pp.1169-1179, 1998.

[8] M. B. Skouson, Q. Guo, and Z. P Liang, “ A bound on mutual information for image registration", IEEE Tran. Medial Imaging, vol. 20, no. 8, pp.843-846, 2001.

[9] F. Maes, A. Collignon, and D. Vandermeulen, Guy Marchal, and Paul Suetens, " Multimodality image registration by maximization of mutual information, IEEE Tran. Medial Imaging, vol. 16, no. 2, pp.187-198, 1997.

[10] W.H. Press, B.P. Flannery, S.A. Teukolsky, and W. T. Vetterling, Numerical Recipes in C. Cambridge Univ. Press, Cambridge, U.K, 1992. 